\title{
SATISFACCIÓN LABORAL: Análisis de las variables predictoras en una muestra de profesionales de la salud, en Atención Especializada, de un área sanitaria de la Comunidad de Madrid
}

\author{
JOB SATISFACTION: Analysis of predictor \\ variables in a sample of healthcare proffessionals, \\ in Specialty Care, of a sanitary area \\ of the Comunidad de Madrid
}

\author{
Antonia Bernat Jiménez \\ Hospital Universitario Clínico "San Carlos". Madrid. España. \\ Pedro Izquierdo Doyagüez \\ Hospital Universitario Clínico "San Carlos". Madrid. España. \\ Lourdes Jiménez Bajo \\ Hospital Universitario Clínico "San Carlos". Madrid. España. \\ Ignacio Bardón Fernández-Pacheco \\ Hospital Universitario Clínico "San Carlos". Madrid. España. \\ $M^{a}$ Nieves Casado Verdejo \\ Hospital Universitario Clínico "San Carlos". Madrid. España. \\ Correspondencia: \\ Antonia Bernat Jiménez \\ C/ Benito Gutiérrez, 24 \\ 28008 Madrid \\ Telef: Trabajo: 913303431 \\ Domicilio: 915432213 \\ Móvil: 636199095 \\ E-mail: abernat.hcsc@salud.madrid.org
}

Resumen

Este trabajo presenta los resultados del estudio de satisfacción laboral realizado a una muestra de 873 trabajadores de la sanidad pública, mediante aplicación de un cuestionario ad hoc, cuya estructura se presenta. A partir de estos resultados, se analizan las variables con capacidad predictiva sobre la satisfacción laboral general en la muestra. El objetivo es la búsqueda de un modelo válido que permita el planteamiento de estrategias preventivas y de intervención en el contexto laboral analizado, como vía para aumentar los niveles de satisfacción y salud laboral.

(Med Segur Trab (Internet) 2009; 55 (217): 49-56)

Palabras clave: Satisfacción laboral, condiciones de trabajo, personal sanitario, modelo predictivo.

Abstract

This work presents the result of a study in job satisfaction performed to a sample of 873 workers of public healthcare by applying an ad hoc questionnaire whose structure will be presented here too. With the support of these results, the variables with predictor potential over the general sample job satisfaction will be analyzed. The main aim of this work is the finding of a valid model that allows the development of 
prevention and intervention strategies in the job context analyzed, as a way to increase job satisfaction and public health.

(Med Segur Trab (Internet) 2009; 55 (217): 49-56)

Key words: Job satisfaction, work conditions, healthcare professionals, predictive model. 


\section{INTRODUCCIÓN}

Desde la teoría de Maslow (1954) y los primeros trabajos de Herzberg (1959), han sido muy numerosas las investigaciones acerca del constructo satisfacción laboral y de las características del lugar de trabajo relacionadas con la satisfacción y, en general, con el bienestar psicológico de los trabajadores (Locke, 1976; Alderfer, 1972; Hackman y OLAM, 1980; Jorge y Schaufeli, 1998; Alonso Morillejo, Pozo y Hernández, 1992).

En las últimas décadas, el creciente interés por la calidad de vida laboral y el impacto del trabajo sobre la salud mental, han vuelto a hacer resurgir el interés y constatar la importancia de esta variable.

Entre los modelos más recientes que relacionan las condiciones de trabajo con la satisfacción laboral y el bienestar de los trabajadores, destacan dos propuestas: el Modelo de Características del Puesto (Hackman y Oldham, 1980) y el Modelo de DemandaControl-Apoyo (Karaseck y Theorell, 1990).

En la actualidad no existe una definición unánimemente aceptada sobre el concepto de satisfacción laboral. Una serie de definiciones hacen referencia a la satisfacción laboral como estado emocional, sentimientos o respuestas afectivas (Locke, 1976; Price y Mueller, 1986), mientras que otro grupo de autores consideran que la satisfacción va más allá de las emociones y la conciben como una actitud general ante el trabajo (Beer, 1964; Harpaz, 1983; Peiró, 1984; Bravo, 1992).

Quizá la concepción más esclarecedora del constructo de satisfacción laboral sea la que la considera como una actitud o conjunto de actitudes desarrolladas por la persona hacia su situación de trabajo. Estas actitudes pueden ir referidas hacia el trabajo en general o hacia facetas específicas del mismo (Fernández Ríos y Sánchez, 1997; Peiró, González-Romá, Bravo y Zurriaga, 1995).

Desde esta aproximación multidimensional, existen numerosas investigaciones que analizan las variables implicadas en la explicación de la satisfacción laboral (el estilo de mando, las relaciones personales, el desarrollo profesional, las retribuciones, etc), obteniendo, en muchos casos, conclusiones no coincidentes, fundamentalmente debido a que determinadas condiciones de trabajo no provocan los mismos efectos en todos los contextos analizados (trabajadores de la salud, docencia, administración, construcción, etc.).

Partiendo de estos planteamientos, ante la inexistencia de un modelo general que asegure que ciertas condiciones vayan a provocar los mismos índices de satisfacción en todos los contextos laborales y, considerando las características especiales de la población objeto de estudio: trabajadores de la sanidad pública en atención especializada, se desarrolló un marco de análisis especifico, sondeando las variables que se relacionan con la satisfacción laboral en nuestro medio y elaborando una herramienta de medida de la misma, con la que se valoró la satisfacción laboral de los trabajadores de sanidad, de Atención Especializada, en un área de salud de la Comunidad de Madrid.

En el presente estudio se analizan las condiciones que inciden en la satisfacción laboral de estos profesionales, en busca de un modelo predictivo válido que permita el planteamiento de intervenciones preventivas en ese contexto.

Las principales hipótesis del estudio son:

1. Que dentro de las condiciones de trabajo analizadas, y considerando las características específicas de la muestra, tendrán un peso importante en la explicación de la satisfacción laboral aquellas variables ligadas a las características del trabajo, al contenido del mismo.

2. Que las variables relacionadas con la organización del trabajo, tendrán un peso importante como variables predictoras de la satisfacción laboral en la muestra. 


\section{OBJETIVO}

La finalidad de este estudio es la obtención de un modelo explicativo de la satisfacción laboral en el personal evaluado. En este sentido, sus principales objetivos son:

1. Conocer los niveles de satisfacción de la población a estudio, así como las variables que la explican.

2. Analizar las variables con capacidad predictiva sobre la satisfacción laboral general, en busca de un modelo válido que permita el planteamiento de estrategias preventivas y de intervención en el contexto laboral evaluado, a fin de aumentar el nivel de satisfacción, como vía para mejorar las condiciones psicosociales y la salud laboral.

\section{MÉTODO}

Se diseña estudio descriptivo transversal. La población a estudio son los profesionales de Atención Especializada de un área sanitaria de la Comunidad de Madrid.

Se desarrolla un marco de análisis especifico, sondeando las variables que se relacionan con la satisfacción laboral en este medio y elaborando una herramienta de medida de la misma.

Para ello, a través de entrevistas individuales y grupales, se realiza una recogida global de información, perfilándose el esqueleto que interpreta la satisfacción en la población diana.

Surgen así 8 agrupaciones de variables homogéneas o dimensiones teóricas de la escala, cuya validez de constructo fue probada, y que constituyen los factores:

$\begin{array}{lll}\text { - FACTOR 1: } & \text { (F1) } & \text { INTERES EN TORNO A LA NATURALEZA DEL } \\ & \text { TRABAJO }\end{array}$

Cada factor está a su vez constituido por varios indicadores. En total, a partir de los 8 factores, surgieron 23 indicadores: Interés del profesional ante el contenido de su trabajo y hacia la organización en que trabaja; Cómo es y qué hace el líder, Trabajo hacia un objetivo común, Cooperación, Habilidades en equipo, Orientación a objetivos, Autonomía, Iniciativa, Respeto por el trabajo de otros, Objetivos estratégicos, Cultura y diseño organizativo, Procesos, Funciones, Desarrollo, Reconocimiento, Investigación, Docencia, Comunicación, Condiciones físicas de trabajo, Relación contractual.

Estos indicadores se reflejan, a su vez, en los ítems del cuestionario.

Se recoge también información sobre variables sociodemográficas: genero, edad, categoría profesional, destino, antigüedad y relación contractual, para la posterior estructuración de la población en colectivos. 
El cuestionario consta de 71 preguntas cerradas, dos preguntas abiertas y una pregunta para la validación. La tipología de respuesta es una escala tipo Likert, siendo 1 la postura más negativa y 4 la más positiva (insatisfactorio / ligeramente insatisfactorio / ligeramente satisfactorio / satisfactorio), asociándose la $5^{\mathrm{a}}$ posibilidad a "no procede".

Los cuestionarios fueron remitidos a todo el personal de Atención Especializada del área, junto con las instrucciones pertinentes y carta informativa acerca de los objetivos del estudio y de la confidencialidad de los datos obtenidos.

Los datos recogidos fueron sometidos a una serie de análisis estadísticos, utilizando para ello el programa informático SPSS.

Para conseguir los objetivos propuestos en el estudio se llevaron a cabo los siguientes tipos de análisis:

1. Estadísticos descriptivos

2. Coeficiente de correlación lineal -r- de Pearson

3. Análisis de regresión, método "paso a paso", en que el análisis elabora sucesivos modelos explicativos, introduciendo en la ecuación de regresión una a una las variables independientes, que van mejorando en cada paso la bondad estadística del modelo realizado.

Todos los análisis estadísticos han sido ponderados.

\section{RESULTADOS}

En el estudio participaron 873 trabajadores (17\% de la población total).

Del total de la muestra, un $72,1 \%$ son mujeres y el $27,9 \%$ restante son hombres. Los datos de edad quedan recogidos en la Tabla 1.

Tabla 1.

\begin{tabular}{lc}
\hline Edad & $\%$ \\
\hline Menos de 25 & 1,7 \\
$26-35$ & 22,7 \\
$36-45$ & 30.7 \\
$46-.55$ & 28.3 \\
$56-65$ & 8.5 \\
Más de 65 & 0.6 \\
No consta & 7.6 \\
\hline
\end{tabular}

Respecto a la variable categoría profesional, están representadas todas las existentes, si bien para resumir, aquí haremos referencia a su agrupación por divisiones. Así, el $22,68 \%$ de la muestra corresponde a personal médico/facultativo, el 50,06\% a personal de enfermería y el 22,68\% restante, a personal de gestión. También se contempló la variable Área laboral/Destino. 
El nivel medio de satisfacción general arroja un valor ligeramente inferior a la media teórica (la variable tiene un rango de 1 a 4 y la media es de 1,85). La dispersión de respuesta es alta en las tres divisiones, sobre todo en la Médica (Tabla 2).

Tabla 2.

\begin{tabular}{lcc}
\hline \multicolumn{3}{c}{ Estadísticos descritivos } \\
\hline Variables & Media & Desviación Típica \\
Satisfacción & 1,8500 & 0,9800 \\
F1 & 2,8853 & 0,6584 \\
F2 & 2,2371 & 0,8730 \\
F3 & 2,4856 & 0,8201 \\
F4 & 3,1903 & 0,4208 \\
F5 & 1,9639 & 0,6450 \\
F6 & 1,8539 & 0,6320 \\
F7 & 1,7596 & 0,5751 \\
F8 & 2,4681 & 0,7889 \\
\hline
\end{tabular}

Como podemos apreciar en la Tabla 2, de todas las dimensiones a estudio son las relativas a la "Profesionalidad responsable" (F4) y a la "Naturaleza del trabajo" (F1), las valoradas más positivamente por la muestra, mientras que el Factor 7, "Planes y políticas" (Formación y Desarrollo, Retribuciones, Investigación, Comunicación) y la "Organización del trabajo" (F6) son los peor considerados, fundamentalmente, dentro de esta, el indicador de "Procesos" que es el que obtiene peores resultados.

Este indicador, comprende aspectos como:

- Definición de procesos y sus responsables

- Planificación y distribución del trabajo

- Funcionamiento de Procedimientos (Inter e Intra Servicio)...

En cuanto al modelo explicativo de la satisfacción general, al analizar el valor predictivo de cada variable por separado, de forma independiente, el mayor poder explicativo corresponde al constructo de "Organización del trabajo" (F6). Tabla 3.

Tabla 3.

\begin{tabular}{ccc}
\hline & Correlaciones & \\
\hline SATISFACCIÓN & \\
$\begin{array}{c}\text { Correlación de } \\
\text { Pearson }\end{array}$ & Satisfacción & 1,000 \\
F1 & 0,388 \\
F2 & 0,522 \\
F3 & 0,457 \\
F4 & 0,334 \\
F5 & 0,506 \\
F6 & 0,577 \\
F7 & 0,541 \\
F8 & 0,233 \\
\hline &
\end{tabular}


El análisis de regresión añade algo más a la simple relación estadística entre cada variable independiente (factores e indicadores) y la variable dependiente (satisfacción), al considerar el impacto interrelacionado de todas las variables. Así, mediante el método de regresión "paso a paso", se elaboran sucesivos modelos explicativos de la satisfacción en la muestra, introduciendo una a una todas las variables independientes que van mejorando en cada paso la bondad estadística del modelo realizado.

Finalmente, el modelo de mayor bondad estadística resultaba de considerar el impacto interrelacionado de los indicadores y estaba formado por 4 de los 23 existentes, explicando un 48,3\% de la varianza total de la satisfacción en el trabajo.

Los resultados de este análisis de regresión, con la satisfacción laboral general como variable dependiente y que incluyó como variables predictoras todos los indicadores, se muestran en la tabla 4: Modelo predictivo.

Tabla 4.

\begin{tabular}{lccc}
\hline Modelo & R & R cuadrado & $\begin{array}{c}\text { R cuadrado } \\
\text { corregida }\end{array}$ \\
\hline $\begin{array}{l}\text { Procesos } \\
\begin{array}{l}\text { Actuación } \\
\text { Líder }\end{array}\end{array}$ &, 622 &, 387 &, 386 \\
$\begin{array}{l}\text { Habilidades } \\
\text { en Equipo }\end{array}$ &, 673 &, 453 &, 451 \\
\begin{tabular}{l} 
Desarrollo \\
\hline
\end{tabular} &, 695 &, 471 &, 468 \\
\hline
\end{tabular}

La relación de estos cuatro indicadores con los distintos factores queda recogida en la Tabla 5.

Tabla 5.

\begin{tabular}{ll}
\hline $\begin{array}{l}\text { Indicadores resultantes } \\
\text { del Modelo Predictivo: }\end{array}$ & Factor del que forman parte \\
\hline Procesos & Organización del trabajo \\
Actuación Líder & Liderazgo - gestión \\
Habilidades en Equipo & Implicación en equipo \\
Desarrollo & Planes y políticas \\
\hline
\end{tabular}

No existen diferencias significativas entre las Divisiones (Médica, Enfermería y Gestión) en cuanto a la interpretación y valoración de los distintos factores como precursores de la satisfacción laboral.

Las variables sociodemográficas se incluyeron en el análisis, no encontrándose diferencias estadísticamente significativas respecto a ninguna de ellas, si bien, arrojaban niveles mayores de satisfacción los trabajadores con antigüedad inferior a cinco años. De igual forma, las mujeres presentaban una valoración de la satisfacción algo inferior a la de los hombres.

\section{DISCUSIÓN Y CONCLUSIONES}

La primera conclusión general es que los conceptos que en la actualidad explican la mayor satisfacción de la población son dos:

- Profesionalidad responsable y

- Naturaleza del trabajo 
- Mientras que los conceptos que explican la menor satisfacción son:

- El Reconocimiento, y

- La Organización del trabajo, en particular, el indicador de Procesos.

La segunda conclusión es que, tomando en consideración todas las valoraciones plasmadas en el cuestionario y tal como indica el modelo predictivo obtenido, la forma de abordar a futuro la satisfacción laboral, considerando el impacto interrelacionado de los indicadores, consiste en actuar, por este orden, sobre:

\section{Procesos $\rightarrow$ ORGANIZACIÓN DEL TRABAJO \\ 2. Actuación del Líder $\rightarrow$ LIDERAZGO-GESTIÓN \\ 3. Habilidades en Equipo $\rightarrow$ TRABAJO EN EQUIPO \\ 4. Desarrollo $\rightarrow$ PLANES Y POLÍTICAS}

Estos resultados vienen a confirmar las hipótesis del estudio, ampliando y clarificando las distintas relaciones entre las diferentes variables, o facetas del trabajo a estudio y la satisfacción laboral en la muestra.

Como conclusión final, resaltar el desequilibrio motivacional entre lo que satisface, ligado a la persona (Profesionalidad responsable y Naturaleza del trabajo/Identificación con el mismo), y lo que no satisface (Organización del trabajo, Reconocimiento), ligado a percepciones sobre lo que la organización aporta para el desarrollo del trabajo.

Por tanto, toda estrategia preventiva o de intervención debe observar ambos enfoques de aproximación, tal como se ve en la validez predictiva.

El colectivo marca, claramente y a futuro, las prioridades actitudinales (Actuación del Líder y Habilidades en Equipo) y organizativas (Procesos y Desarrollo) orientadas a la consecución de mayores niveles de satisfacción laboral en el mismo.

\section{REFERENCIAS}

1. Acker, G.M. (2004). The effect of organizational conditions on job satisfaction and intention to leave among social workers in mental health. Community Mental Health, 40. (1), 65-73.

2. Alonso Morillejo, E. y Pozo, C. (2001). Análisis de los factores psicosociales de riesgo en los profesionales dedicados al cuidado de la salud. Revista de Psicología del Trabajo y de las Organizaciones, 17 (3), 273-293.

3. Karasek, R. y Theorell, T. (1990). Healthy work, productivity, and the reconstruction of working life. Nueva York: Basic Books.

4. Locke, E. A. (1976) The nature and causes of job satisfation. En M. D. Dunnette (Eds.). Handbook of Industrial and Organization Psychology. Nueva York: John Wiley \& Sons.

5. Meliá, J. L. y Peiró, J. M. (1989). Cuestionario de Satisfacción Laboral S10/12. Estructura factorial, fiabilidad y validez. Psicología del Trabajo y de las Organizaciones, 11, 179-185.

6. Peiró, J.M., González-Romá, V.,Bravo, M.J. y Zurriaga, R. (1995). La medida de la satisfacción laboral. Ansiedad y Estrés, 1 (2-3), 231-253.

7. Peiró, J.M. y Prieto, F. (1996, eds.): La actividad laboral en su contexto. Aspectos psicosociales del trabajo. Tratado de Psicología del trabajo. (Madrid. Síntesis Psicología.

8. Pozo, C., Alonso, E. Hernández, S. y Martos, M.J. (1995). Determinantes de la satisfacción laboral en trabajadores de la administración pública: El valor de las relaciones interpersonales en el lugar de trabajo. Ansiedad y Estrés, 11 (2-3), 247-264. 